\title{
Corrected QT Interval in Systemic Sclerosis Patients
}

\author{
Alexandru Caraba ${ }^{1 *}$, Andreea Munteanu ${ }^{1}$, Flavia Corina Babalic ${ }^{1}$ and Mihaela Nicolin ${ }^{2}$ \\ ${ }^{1}$ Department of Internal Medicine, Division of Rheumatology, Victor Babeș University of Medicine and Pharmacy, Romania \\ ${ }^{2}$ Department of Cardiology, Victor Popescu Emergency Clinical Military Hospital Timisoara, Romania
}

*Corresponding author: Alexandru Caraba, Department of Internal Medicine, Division of Rheumatology, Victor Babeș University of Medicine and Pharmacy, Timișoara, Romania.

Received Date: August 29, 2019

Published Date: September 09, 2019

\begin{abstract}
Introduction: Cardiac involvement in patients with systemic sclerosis represents an important cause of morbidity and mortality. Electrocardiographic abnormal findings are identified in 25-75\% of SSc patients, being considered an independent predictor of mortality. In SSc, even without cardiac symptoms, QT and corrected QT (QTc) intervals appear prolonged, which can lead to life-threatening tachyarrhythmias. The aim of this study was to assess the QTc interval in SSc patients, and, on the other hand, the correlations between QTc and nailfold capillary findings in these patients.
\end{abstract}

Material and methods: This case control study was performed on a group of 22 patients with SSc, who fulfilled the 2013 ACR/EULAR Classification Criteria for Systemic Sclerosis and 22 healthy subjects, matched for age and gender, as controls. In all the SSc patients and controls were performed: 12-lead standard electrocardiographic recordings and nailfold capillaroscopy. QTc interval and nailfold capillaries density were recorded in SSc patients and controls. In SSc were determined: antinuclear antibodies, anti-topoisomerase I, anti-centromere and anti-RNA polymerase III antibodies, too. Data are presented as mean \pm standard deviation. Statistical analyses were performed using the Student's t-test, ANOVA test, and the Pearson's correlation. Differences were considered statistically significant at the value of $\mathrm{p}<0.05$.

Results: The values of QTc interval were prolonged in SSc group than in controls ( $p<0.01)$. These values of QTc interval increased with the severity of the nailfold capillaroscopic pattern, the differences having statistical significance $(p<0.001)$. It was demonstrated a statistically significant negative correlation between the values of QTc intervals and nailfold capillaries density, this correlation being stronger with the increase of the severity of nailfold capillaroscopic pattern.

Conclusion: SSc patients present prolonged QTc interval, even they are without any cardiac symptoms, requiring the ambulatory 24-hour ECG monitoring in order to identify ventricular arrhythmias and initiate appropriate therapy.

Keywords: Corrected QT interval; Nailfold capillaroscopy; Systemic sclerosis

\section{Introduction}

Systemic sclerosis (SSc) is a chronic disorder, characterized by autoimmunity, inflammation, functional and then structural abnormalities of micro vessels and, finally, widespread interstitial and vascular fibrosis involving skin and internal organs [1]. Based on clinical features and the presence of specific SSc-related autoantibodies, the following forms of SSc have been described: limited SSc (lcSSc), diffuse SSc (dcSSc) and SSc without skin involvement [2].

Cardiac involvement in SSc can be primary, direct consequence of this disease or secondary, associated with SSc pulmonary hypertension or renal crisis [3]. The clinical features of SSc heart involvement are highly variable, from silent forms to heart failure. Based on this fact, the prevalence of SSc cardiac involvement varies greatly, from $10 \%$ to $50 \%$, depending on the diagnostic method used (clinical exam, electrocardiography, cardiac ultrasonography, cardiac magnetic resonance imaging) [4]. The rapid skin thickness progression is associated with higher cardiac involvement. Cardiac causes represent $20 \%$ to $36 \%$ of deaths associated with SSc. Several mechanisms are involved in SSc heart disease: microvascular alterations, myocardial inflammation, fibrosis and autonomic dysfunction $[1,2,5]$.

Electrocardiographic abnormal findings are identified in $25-75 \%$ of SSc patients, being represented by: atrial and ventricular tachyarrhythmias, conduction abnormalities and bradyarrhythmias. They are considered to be an independent predictor of mortality [6]. In SSc, even without cardiac symptoms, QT and corrected QT (QTc) intervals appear prolonged, which can lead to life-threatening tachyarrhythmias [7]. 
QT interval, measured from the beginning of the QRS complex to the end of the $\mathrm{T}$ wave, represents the time required for all ventricular depolarization and repolarization processes to occur. It depends on many physiologic and pathologic factors, including heart rate, which plays a major role. Several methods have been used to correct the QT interval, all of which consider the heart rate, generating corrected QT (QTc) [8].

The aim of this study was to assess the QTc interval in SSc patients, and, on the other hand, the correlations between QTc and nailfold capillary findings in these patients.

\section{Materials and Methods}

\section{Patients}

This case control study was performed on a group of 22 patients with SSc without cardiac symptoms and 22 healthy subjects, matched for age and gender, as controls. All patients fulfilled the 2013 ACR/EULAR Classification Criteria for Systemic Sclerosis [9]. Exclusion criteria were represented by: overlap syndromes, overt cardiac diseases unrelated with SSc, tachyarrhythmias with heart rate higher than 90 beats/minute, pre-existing branch blocks, uncontrolled systemic hypertension, pulmonary hypertension, right ventricular dysfunction, diabetes mellitus, chronic kidney disease, current smokers, pregnancy or breastfeeding women, treatment with drugs which prolonged QT interval. All the patients gave their informed consent. The study was approved by the Ethics Committee of "Victor Babeș" University of Medicine and Pharmacy, Timişoara, Romania. This study respects the Declaration of Helsinki.

\section{Methods}

12-lead standard electrocardiographic recordings (recording speed of $25 \mathrm{~mm} / \mathrm{sec}$, voltage $10 \mathrm{~mm} / \mathrm{mV}$ ) using BTL-08 SD3 equipment were performed in all patients and controls. QT interval was recorded in all patients and controls; then, using Bazzett's formula, QTc interval was determined. Normal values of QTc interval were below $440 \mathrm{msec}$ [8].

The density of nailfold capillaries/mm was determined by nailfold capillaroscopy (USB Digital Microscope, 2.0Mega Pixel Digital Camera). Before this procedure, the patients and controls took place in a room with a stable temperature of $20-22^{\circ} \mathrm{C}$ for at least 15 minutes, in order to avoid capillaries vasoconstriction, which can induce false positivity for avascular areas. The $2 \mathrm{nd}, 3 \mathrm{rd}, 4$ th and 5 th fingers of both hands were examined. Giant capillaries, capillaries hemorrhages, avascular areas, ramified/bushy capillaries, and capillary architecture were the recorded nailfold capillaroscopic parameters. Patients with SSc may develop three capillaroscopic patterns defined as: early, active, and late [10]. Nailfold capillaries density/mm was the parameter used in the statistical analysis.

Antinuclear antibodies, anti-topoisomerase, anti-centromere, and anti-RNA polymerase III antibodies were determined using indirect immunofluorescence (HELMED).

\section{Statistical analysis}

Data are presented as mean \pm standard deviation. Statistical analyses were performed using the Student's t-test, ANOVA test, and the Pearson's correlation. Differences were considered statistically significant at the value of $\mathrm{p}<0.05$.

\section{Results}

Baseline demographic data of SSc patients and controls are presented in the Table 1.

Table 1: Demographic data in SSc patients and controls.

\begin{tabular}{|c|c|c|}
\hline \multirow[t]{2}{*}{ Parameter } & \multicolumn{2}{|c|}{ Value (Mean \pm Standard Deviation) } \\
\hline & SSc Patients & Controls \\
\hline $\begin{array}{c}\text { Sex }[\mathrm{n}(\%)] \\
\text { Males } \\
\text { Females }\end{array}$ & $\begin{array}{c}22 \\
6(27.27 \%) \\
16(72.72 \%)\end{array}$ & $\begin{array}{c}22 \\
6(27.27 \%)\end{array}$ \\
\hline Mean age (years) & $50 \pm 4.55$ & $51.24 \pm 4.72$ \\
\hline $\begin{array}{l}\text { Mean length of SSc } \\
\text { evolution (years) }\end{array}$ & $5.36 \pm 3.18$ & - \\
\hline $\begin{array}{c}\text { Mean length } \\
\text { of Raynaud's } \\
\text { phenomenon } \\
\text { evolution (years) }\end{array}$ & $8.36 \pm 3.68$ & - \\
\hline $\begin{array}{l}\text { The drugs used by } \\
\text { the SSc patients } \\
\text { in the moment of } \\
\text { investigation }\end{array}$ & $\begin{array}{c}\text { Azathioprine } \\
\text { (14 patients) } \\
\text { Cyclophosphamide } \\
\text { (6 patients) } \\
\text { Methotrexate (2 } \\
\text { patients) }\end{array}$ & - \\
\hline
\end{tabular}

Among the SSc patients, 19 had diffuse cutaneous SSc, whereas 3 patients had the limited form of the disease. Raynaud's phenomenon was present in all cases.

Antinuclear antibodies were demonstrated in all patients. Anti-topoisomerase I antibodies were identified in 12 SSc patients, anti-RNA polymerase III antibodies in 7 SSc patients, whereas anticentromere antibodies in 3 patients.

The density of nailfold capillaries was reduced in SSc patients than in controls $(p<0.001)$. The values of QTc interval were prolonged in SSc group than in controls, the difference being statistically significant $(\mathrm{p}<0.01)$ (Table 2 ).

Table 2: Parameters assessed in SSc patients and controls.

\begin{tabular}{|c|c|c|c|}
\hline Parameter & SSc Patients & Controls & p \\
\hline QTc $(\mathrm{msec})$ & $455.31 \pm 44.60$ & $420.68 \pm 19.75$ & $<0.01$ \\
\hline $\begin{array}{c}\text { Capillaries } \\
\text { density } / \mathrm{mm}\end{array}$ & $8.06 \pm 2.45$ & $11.54 \pm 1.04$ & $<0.001$ \\
\hline
\end{tabular}

Table 3: QTc interval values in SSc patients.

\begin{tabular}{|c|c|c|c|c|}
\hline \multirow{2}{*}{ Parameter } & \multicolumn{3}{|c|}{ Nailfold Capillaroscopic Pattern } & p \\
\cline { 2 - 4 } & Early & Active & Late & \multirow{2}{*}{} \\
\hline $\begin{array}{c}\text { Number of } \\
\text { patients }\end{array}$ & 6 & 9 & 7 & \\
\hline $\begin{array}{c}\text { Capillaries } \\
\text { density/ } \\
\text { mm }\end{array}$ & $10.85 \pm 0.56$ & $8.42 \pm 0.94$ & $5.18 \pm 1.34$ & $<0.001$ \\
\hline QTc (msec) & $\begin{array}{c}411.83 \pm \\
18.46\end{array}$ & $\begin{array}{c}449.77 \pm \\
21.06\end{array}$ & $\begin{array}{c}499.71 \pm \\
43.01\end{array}$ & $<0.001$ \\
\hline
\end{tabular}

Based on Cutolo's capillaroscopic patterns [10], the studied SSc patients were classified as: early ( 6 patients), active ( 9 patients), and late (7 patients) capillaroscopic patterns. The data analysis 
revealed that the values of QTc interval increased with the severity of the capillaroscopic pattern, the differences having statistical significance (Table 3).

Statistical analysis highlighted the presence of statistically significant correlations between the values of QTc intervals and nailfold capillaries density and, on the other hand, with the mean length of SSc evolution (Table 4).

Table 4: Correlations between QTc values and demographic data.

\begin{tabular}{|c|c|c|}
\hline $\begin{array}{c}\text { Correlation Between QTc and Nailfold } \\
\text { Capillaries Density: }\end{array}$ & $\mathbf{r}$ & $\mathbf{p}$ \\
\hline Mean age & -0.1272 & 0.57 \\
\hline Mean length of SSc evolution & 0.4408 & $<0.05$ \\
\hline $\begin{array}{c}\text { Mean length of Raynaud's phenomenon } \\
\text { evolution }\end{array}$ & -0.0036 & 0.98 \\
\hline Nailfold capillaries density/mm & -0.8905 & $<0.001$ \\
\hline
\end{tabular}

The progression of SSc microangiopathy, proved by nailfold capillaroscopic pattern, was associated with prolongation of the QTc interval. If at the beginning of the SSc (revealed by early capillaroscopic pattern), the correlation between QTc interval and nailfold capillaries density did not exist, as the disease worsened (active and late capillaroscopic patterns), the negative correlations become more and more significant (Table 5).

Table 5: Correlations between QTc values and capillaroscopic patterns.

\begin{tabular}{|c|c|c|}
\hline $\begin{array}{c}\text { Correlation Between QTc and } \\
\text { Nailfold Capillaries Density/mm: }\end{array}$ & $\mathbf{r}$ & $\mathbf{p}$ \\
\hline Early pattern & 0.0887 & 0.69 \\
\hline Active pattern & -0.6963 & $<0.001$ \\
\hline Late pattern & -0.8432 & $<0.001$ \\
\hline
\end{tabular}

Regarding the QTc interval duration according to the SScrelated antibodies profile, it was found that the presence of RNApolymerase III antibodies was associated with the longest duration of the QTc interval (anti-centromere antibodies: 417.66 \pm 37.85 msec, anti-topoisomerase I antibodies: $449.91 \pm 40.97 \mathrm{msec}$, antiRNA polymerase III antibodies: $480.71 \pm 43.43 \mathrm{msec}, \mathrm{p}<0.001$ ). But it should be noted that 4 of the 7 patients with anti-RNA polymerase III antibodies had late capillaroscopic pattern.

\section{Discussion}

The present study performed on patients with SSc, showed that in these patients, the QTc interval was longer than in controls. Moreover, there was a negative correlation between the QTc interval values and the nailfold capillaries density. In other words, the progression of SSc microangiopathy, proved by nailfold capillaroscopic pattern, was associated with QTc interval prolongation. If at the beginning of the SSc (revealed by early capillaroscopic pattern), this correlation did not exist, as the disease worsened (active and late capillaroscopic patterns), the correlation become more and more significant $(r=-0.6963, p<0.001$ for the patients with active capillaroscopic pattern, respective $r=-0.8432$, $\mathrm{p}<0.001$ for the patients with late capillaroscopic pattern).

SSc microangiopathy is present in all organs of these patients. Nailfold capillaroscopy has become a useful tool in staging of microcirculation involvement in SSc patients, offering details about the disease severity and degree of vascularization in patients with it $[10,11]$. Cutolo et al. defined three evolution patterns of microvascular involvement in SSc patients, named as: early (few giant capillaries, few capillary microhemorrhages, no evident loss of capillaries, and a relatively well-preserved capillary distribution), active (frequent giant capillaries, frequent capillary microhemorrhages, moderate loss of capillaries, absence of or mildly ramified capillaries with slight disorganization of the capillary architecture), and late (irregular enlargement of the capillaries, almost absent giant capillaries and microhemorrhages, severe loss of capillaries with extensive avascular areas, ramified/ bushy capillaries, and intense disorganization of the normal capillary array), having a role in assessing the appearance and progression of sclerodermic microangiopathy $[12,13]$.

Pathogenesis of SSc heart disease is related to cardiac microangiopathy (without epicardial vessels involvement), which drives to ischemia, inflammation and fibrosis [3]. Recurrent myocardial ischemia, induced by microvascular dysfunction (myocardial Raynaud's phenomenon), generates patchy myocardial fibrosis. This myocardial fibrosis is identified in $50 \%$ to $70 \%$ of SSc patients, generating prolongation the time required for all ventricular depolarization and repolarization processes to occur. The electrocardiographic sign is represented by the prolonged QTc interval $[14,15]$. But beside myocardial fibrosis, autonomic dysfunction contributes to QTc prolongation, too [6].

Wei et al. showed that the QTc interval prolongation represented an independent risk factor for cardiac mortality and sudden death [16]. Arrhythmias may be associated with poor outcome and represent $6 \%$ of the overall causes of death in SSc patients [17]. According to Vacca and el, cardiac arrhythmias are identified in 25$75 \%$ of SSc patients [7].

Prolonged QTc interval was identified in SSc patients even without clinical signs of miocardial involvement [18].

Studying 72 SSc patients and 64 controls, Morelli et al. showed that the SSc patients presented significantly longer QTc interval than the controls $(\mathrm{p}=0.0016)$ [19]. Thirty-eight patients with SSc (19 patients with dcSSc and 19 with the lcSSc) and 17 healthy controls were studied by Sgreccia et al. The authors identified that the SSc patients had increased QTc interval, QT dispersion, and QTc dispersion [20]. Bellando-Randone et al. identified that the SSc patients presented prolonged QTc interval, this situation being associated with increased risk of sudden deaths [21]. In the study performed by Massie et al., prolonged QTc interval was common in SSc patients, being associated with anti-RNA polymerase III antibodies, longer disease duration, and greater disease severity [22]. In the present study, the patients with anti-RNA polymerase III antibodies presented the longest duration of QTc interval; but $57.14 \%$ of them had late capillaroscopic pattern. Rosato et al. studying twenty SSc patients, showed that the values of QTc intervals were significantly increased in SSc patients than controls ( $447 \mathrm{msec}$ vs $386 \mathrm{msec})(\mathrm{p}<0.0001)$. The authors revealed that the values of the QT intervals increased with the severity of the nailfold capillaroscopic pattern: early pattern: $425 \mathrm{msec}$ (421-454), active pattern 437 msec (416-467), and late pattern $471 \mathrm{msec}$ (445-566) $(p<0.01)$. No correlations were found between the values of QTc 
interval and SSc subset or duration, but the presence of digital ulcers and high modified Rodnan total skin score were correlated with the values of this interval [23]. Similar findings regarding to the values of QTc intervals and nailfold capillaroscopic pattern were identified in the present study. One study showed that in dcSSc patients, QTc interval was significantly prolonged than in lcSSc patients [24], but it has not been confirmed by other studies. Another study performed on $65 \mathrm{SSc}$ patients and 63 control subjects showed that the QTc intervals were significantly higher in the former $(\mathrm{p}<0.01)$, without any difference between patients with dcSSc and lcSSc [15]

QTc interval prolongation in SSc patients with late capillaroscopic pattern is associated with a high risk of developing life-threatening ventricular arrhythmias [23]. If the prolonged QTc interval is demonstrated, then the ambulatory 24-hour ECG monitoring is required to identify ventricular arrhythmias and initiate appropriate therapy.

The relatively small number of SSc patients is one of the limits of this study. Large-scale prospective studies are required to identify the risk factors of life-threatening ventricular arrhythmias in SSc patients with prolonged QTc interval.

\section{Conclusion}

SSc patients present prolonged QTc interval, even they are without any cardiac symptoms. In order to identify the SSc heart disease from its earliest stages, it is advisable to perform the electrocardiogram once a year, especially in patients with significant nailfold capillaries findings.

\section{Acknowledgment}

None.

\section{Conflict of Interest}

The authors declared no conflicts of interest.

\section{References}

1. Varga J (2017) Etiology and Pathogenesis of Scleroderma. In: Firestein GS, Budd RC, Gabriel SE, McInnes IB, O'Dell JR. Kelley\&Firestein's Textbook of Rheumatology, $10^{\text {th }}$ Edn, Elsevier, Philadelphia, pp. 14001423.

2. Wigley FM, Boin F (2017) Clinical Features and Treatment of Scleroderma. In: Firestein GS, Budd RC, Gabriel SE, McInnes IB, O'Dell JR. Kelley\&Firestein's Textbook of Rheumatology, $10^{\text {th }}$ Edn, Elsevier, Philadelphia, pp. 1424-1460.

3. Rubini G, Vinci F, Farina L, Riccieri V, Sciarra I, et al. (2017) Electrocardiographic and Echocardiographic findings and their Relationships in Patients with Systemic Sclerosis. J Autoimmun Res 4(1): 1017

4. Parks JL, Taylor MH, Parks LP, Silver RM (2014) Systemic sclerosis and the heart. Rheum Dis Clin North Am 40(1): 87-102.
5. Vacca A (2017) Systemic sclerosis. In: Nussinovitch U (Ed). The Heart in Rheumatic, Autoimmune and Inflammatory Diseases. Elsevier, pp.301326.

6. Stefanovic Neskovic J, Ristic A, Petronijevic M, Neskovic B, Gudelj O, et al (2017) Electrocardiographic findings in systemic sclerosis. Ser J Exp Clin Res 1-1.

7. Vacca A, Meune C, Gordon J, Chung L, Proudman S, et al. (2014) Cardiac arrhythmias and conduction defects in systemic sclerosis. Rheumatology 53(7): 1172-1177.

8. Goldberger AL, Goldberger ZD, Shvilkin A (2018) Goldberger's Clinical Electrocardiography. A Simplified Approach, $9^{\text {th }}$ Edn, Elsevier.

9. Van den Hoogen F, Khanna D, Fransen J, Johnson SR, Baron M, et al. (2013) 2013 Classification Criteria for Systemic Sclerosis: an American College of Rheumatology/European League against Rheumatism collaborative initiative. Ann Rheum Dis 72(11): 1747-1755.

10. Cutolo M, Sulli A, Secchi ME, Paolino S, Pizzorni C (2006) Nailfold capillaroscopy is useful for the diagnosis and follow-up of autoimmune rheumatic diseases. A future tool for the analysis of microvascular heart involvement? Rheumatology 45 (suppl 4): iv43-iv46.

11. Souza EJ, Kayser C (2015) Nailfold capillaroscopy: relevance to the practice of rheumatology. Rev Bras Reumatol 55(3): 264-271.

12. Cutolo M,Sulli A, Pizzorni C, Accardo S(2000) Nailfold videocapillaroscopy assessment of microvascular damage in systemic sclerosis. J Rheumatol 27(1): 155-160.

13. Cutolo M (2011) Atlas of capillaroscopy in rheumatic diseases. (1 $1^{\text {st }}$ Edn) Milano: Elsevier, p. 208.

14. Morelli S, Piccirillo G, Fimognari F, Sgreccia A, Ferrante L, et al. (1996) Twenty-four-hour heart rate variability in systemic sclerosis. J Rheumatol 23(4): 643-645.

15. Yayla C, Yayla EM, Yayla KG, Ilgen U, Akboga MK ,et al. (2016) The assessment of Tp-e interval and Tp-e/QT ratio in patients with systemic sclerosis. Arch Rheumatol 31(2): 139-144.

16. Wei K, Dorian P, Newman D, Langer A (1995) Association between QT dispersion and autonomic dysfunction in patients with diabetes mellitus. J Am Coll Cardiol 26(4): 859-863.

17. Tyndall AJ, Bannert B, Vonk M, Airo P, Cozzi F, et al. (2010) Causes and risk factors for death in systemic sclerosis: a study from the EULAR Scleroderma Trials and Research (EUSTAR) database. Ann Rheum Dis 69(10): 1809-1815

18. Gigante A, Rosato E, Liberatori M, Sardo L, Di Paolo M, et al. (2016) In systemic sclerosis prolonged QTc interval is associated with reduced exercise tolerance. Int J Cardiol 203: 570-572.

19. Morelli S, Sgreccia A, Ferrante L, Barbieri C, Bernardo ML, et al. (1996) Relationships between electrocardiographic and echocardiographic findings in systemic sclerosis (scleroderma). Int J Cardiol 57(2): 151160.

20. Sgreccia A, Morelli S, Ferrante L, Perrone C, De Marzio P, et al. (1998) QT interval and QT dispersion in systemic sclerosis (scleroderma). J Intern Med 243(2): 127-132.

21. Bellando-Randone S, Guiducci S, Matucci-Cerinic M (2012) Very early diagnosis of systemic sclerosis. Pol Arch Med Wewn 122 (Suppl 1): 1823. 